\title{
Ising model with long range correlated disorder on hierarchical lattices
}

\author{
Roberto F. S. Andrade ${ }^{1}$ and Daniel Cason ${ }^{2}$ \\ ${ }^{1}$ Instituto de Física, Universidade Federal da Bahia, CEP 40210-210 Salvador, BA, Brazil \\ ${ }^{2}$ Instituto de Matemática, Universidade Federal da Bahia, CEP 40210-210 Salvador, BA, Brazil \\ (Received 6 September 2009; revised manuscript received 29 November 2009; published 12 January 2010)

\begin{abstract}
A next-neighbor Ising model with disordered but long range correlated coupling constants is investigated. The model is built on a hierarchical lattice and the correlation strength depends on a tuning parameter $\alpha$. The results are obtained within a transfer-matrix framework, which allows for the evaluation of the properties of individual samples. Collective behavior is computed by averaging over a large number of independent realizations. The dependence of the thermodynamic and magnetic functions with respect to the temperature is investigated for each value of $\alpha$. Phase diagrams in the $(\alpha, T)$ plane are constructed for two distinct versions of the model, indicating the existence of regions of paramagnetic and ordered phases. Critical values $\alpha_{c}$, below which the system always assumes the paramagnetic phase, are found for both versions.
\end{abstract}

DOI: 10.1103/PhysRevB.81.014204

PACS number(s): 05.50.+q, 05.70.Fh, 75.10.Pq

\section{INTRODUCTION}

Effects of disorder in physical systems have attracted the attention of the physics community for a long time..$^{1-3}$ Several theoretical approaches have been proposed to describe its ubiquitous presence in natural or devised materials. Most of them consider parameter-dependent models in which a continuous transition from an ordered situation to a fully random one can be followed by tuning a control parameter. A very elegant way to introduce long range correlated disorder in the coupling constants of multicomponent homogeneous systems has been proposed some years ago, ${ }^{4}$ in the context of an electron model to describe the metal-insulator transition. In this specific domain, several different approaches to investigate disordered systems have been proposed,, 56 all of which can be extended or adapted to magnetic systems.

The basic idea of the model proposed in Ref. 4 is to introduce $N$-correlated random coupling constants $\epsilon_{x}, x$ $\in[1, N]$, by imposing that the spectral density $S(k) \sim k^{-\alpha}$. $S(k)$ corresponds the Fourier transform of the correlation function $C(y)=\left\langle\epsilon_{x} \epsilon_{x+y}\right\rangle$ between two coupling constants separated by $y$ intermediate steps. A set of $N$ such constants is generated by

$$
\epsilon_{x}=\sum_{k=1}^{N / 2}\left[\frac{1}{k^{\alpha}}\left(\frac{2 \pi}{N}\right)^{1-\alpha}\right]^{1 / 2} \cos \left(\frac{2 \pi k x}{N}+\phi_{k}\right),
$$

where $\phi_{k}$ is an equally distributed random phase oven the $[0,2 \pi]$ interval. If we set $\alpha=0$, Eq. (1) correctly assigns a completely uncorrelated distribution of events $\left\langle\epsilon_{x} \epsilon_{x+y}\right\rangle$ $=\epsilon^{2} \delta_{y, 0},{ }^{4,7}$ as can be easily seen by just summing the roots of 1 in the complex plane. In the limit $\alpha \rightarrow \infty, \epsilon_{x}$ is dominated by the first term of the series. Besides its original use to address the question of electronic tight-binding state localization, the distribution, Eq. (1), has been used to investigate the properties of several other physical systems, such as spin waves in magnetic systems, ${ }^{8}$ disordered Heisenberg chains, ${ }^{9}$ normal modes in harmonic chains, ${ }^{10}$ etc. For such systems, $P(x)$ helps understanding the continuous transition between the fully uncorrelated situation to the rather ordered situation in the limit of large $\alpha$.
In the current work, we consider a disordered model formed by Ising spins on the diamond hierarchical lattice (DHL), ${ }^{11,12}$ where $J_{i j}$, the random nearest-neighbor coupling constants between spins $i$ and $j$, are chosen with the help of Eq. (1). It is well known ${ }^{13}$ that the uniform version of this model $\left[J_{i j}=J, \quad \forall(i j)\right]$ undergoes a transition from the paramagnetic to the ferromagnetic phases at a finite temperature $T_{c}$. The value of $T_{c}$ does not depend whether the coupling is ferromagnetic $(\mathrm{F}, J>0)$ or antiferromagnetic $(\mathrm{AF}, J<0)$. On the other hand, the disordered version of the model displays a different behavior. In such case, where each $J_{i j}$ is randomly chosen with equal probability to be $+|J|$ or $-|J|$, the model does not present any critical behavior, being observed in the paramagnetic state for any value of $T>0$. Although DHL's provide crude approximations for the critical behavior of ordered models on Euclidian lattices, the results for disordered models on the two different lattices are much closer. This justifies the widespread use of DHL's in the investigation of such models.

The motivation of this work is to understand how the system behaves when we go, in a smooth way, from the model defined by a completely disordered distribution of $\mathrm{F}$ and AF couplings to the model constituted by uniform coupling constants. This can be accomplished by introducing correlation between the coupling constants. Since the value of $\alpha$ in Eq. (1) defines the correlation strength, it becomes important to understand how its value is related to the emergence of critical behavior and the presence of an ordered phase in the low-temperature region. To this purpose we let, in Eq. (1), $\operatorname{sgn}\left(\epsilon_{x}\right) \rightarrow J_{x} \rightarrow J_{i j}$, where the last step of the process will be detailed discussed in the next section.

Let us mention that many numerical simulations on disordered magnetic systems, indicating that a phase transition between the paramagnetic and the low-temperature spinglass state occurs only for systems with dimension larger than the critical value $2.5 .{ }^{14}$ Although this value primarily refers to the usual Euclidian dimension, all results obtained so far show that the same is valid to systems that are better described by noninteger fractal dimensions $\left(d_{f}\right)$ : for the DHL we consider in this work, which is characterized by $d_{f}=2$, all attempts to characterize a spin-glass phase have failed, ${ }^{15}$ and the paramagnetic state is observed for all values of the tem- 
perature $T$. In any case, as the quoted results do not hint that a spin-glass phase should appear in the considered model, the ordered phase is likely to be of ferromagnetic nature.

The current work is related to previous investigations of the of random Ising systems on DHL's, ${ }^{16}$ where the nearestneighbor coupling constants $J_{i j}= \pm J$ are chosen according to the probability density $P(x)=\delta(x-J) \mathbf{p}+\delta(x+J)(1-\mathbf{p})$, where $\mathbf{p} \in[0,1]$. The value $\mathbf{p}=0.5$ corresponds to a fully random system, with $\mathrm{F}$ and $\mathrm{AF} \pm J$ coupling constants appearing with equal probability, while $\mathbf{p}=1$ reproduces the $\mathrm{F}$ model. On varying the value of $\mathbf{p}$ from 0.5 to 1 , it was possible to show that when the number of $\mathbf{p}$ reaches a critical value $\mathbf{p}=\mathbf{p}_{\mathbf{N}}$ $<\mathbf{1}$, the ratio of the number of $\mathrm{F}$ to $\mathrm{AF}$ bonds in the model is sufficient to produce a magnetic ordering at $T>0$. Below $\mathbf{p}_{\mathbf{N}}$, no ferromagnetic ordering is observed. These results further indicate this model satisfy several properties related to the so-called Nishimori line (NL) (Refs. 17-19) in the $(\mathbf{p}, T)$ plane, as the point of coordinates $\left(\mathbf{p}_{\mathbf{N}}, T_{N}\right)$ lies on the NL. ${ }^{20}$

In the model investigated herein, the parameter $\alpha$ plays a similar role to that of $\mathbf{p}$. Our results will show that $\alpha=0$ corresponds to $\mathbf{p}=0.5$ and, if $\alpha$ is larger than a given threshold value, the system will present a magnetic ordering in the low-temperature phase. Therefore, our results will be summarized by phase diagrams in the $(\alpha, T)$ plane, which assign the regions of ferromagnetic and paramagnetic phases.

The paper is organized as follows: in Sec. II, we introduce two models that distinguish themselves by the way along which the correlated coupling constants are distributed over the DHL. We also briefly recall the major features of the transfer-matrix (TM) method used to obtain the thermodynamic and magnetic properties of the model. In Sec. III, we present the results for two models, discussing the thermodynamic behavior functions and phase diagrams in the $(\alpha, T)$ plane. Section IV presents further remarks and perspectives for future works.

\section{MODELS AND METHOD}

Both models are described by a formal Hamiltonian

$$
H=-\sum_{(i j)} J_{i j} \sigma_{i} \sigma_{j}
$$

where $\sigma_{i}= \pm 1$ are Ising variables and the sum is carried over pairs of nearest neighbors on the DHL.

The DHL is built along the usual way, starting with a line segment linking the two root sites $\left(r_{1}, r_{2}\right)$ in the first generation $G=1$. For any value of $G>1$, the lattice is obtained by replacing each bond of the previous generation $G-1$ by a set of $p=2$ parallel branches, each one with $b-1=1$ inner site (see Fig. 1). The self-similar graph resulting from this construct has a fractal dimension $d_{f}=\log p b / \log b .{ }^{21}$ Other values for $p$ and $b$ can be selected but, in this work, we restrict ourselves to the quoted values of $p$ and $b$, so that $d_{f}=2$.

We consider two different ways to insert the correlated sequence of coupling constants into the lattice. In the longitudinal $\mathbf{L}$ model, the $J_{x}$ values are sequentially distributed along each of the $2^{G}$ distinct paths connecting the two root sites, after the lattice is grown up to a given $G$ [see Fig. 1(a)]. By contrast, Fig. 1(b) shows the coiled model $(\mathbf{C})$, where
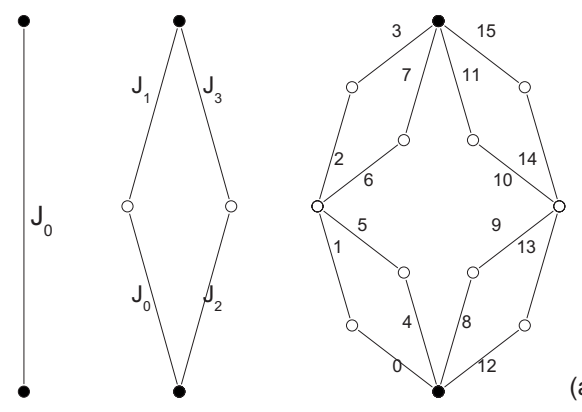

(a)
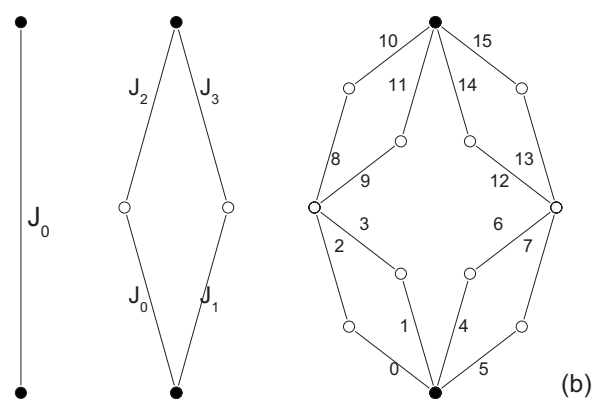

FIG. 1. First three steps of construction of models (a) $\mathbf{L}$ and (b) C on a DHL. The constants $J_{x}$ are chosen according to PDF (1). In (a), four distinct paths are ordered from left to right, consisting, respectively, of constants $J_{x}$ with $x \in[0,3],[4,7],[8,11]$, and $[12,15]$. In (b), the constants are sequentially assigned to the bonds at each new generation, without relation to the distribution in the previous generations.

values of $J_{x}$ are sequentially distributed along with the construction of the lattice. In this construction, each new generation sample is obtained by putting together four samples of the previous generation.

The results are obtained within a TM framework introduced in previous works to investigate the properties of disordered Ising systems with both symmetric and asymmetric distributions $P(J)$ of $\mathrm{F}$ and $\mathrm{AF}$ bonds. ${ }^{15,16}$ Within this method, a set of individual realizations of the model is considered, each of which is obtained by choosing different seed values of the pseudorandom number generator that feeds the distribution $P(J)$. At the end of the process, the evaluated thermodynamic functions for each individual sample $i$ $\in\left[1, N_{t}\right]$ (free energy $f_{i}$, entropy $s_{i}$, specific heat $c_{i}$, magnetization $m_{i}$, and correlation length $\xi_{i}$ ) are averaged out, providing the most important properties of the system. The values of the critical temperature $T_{c}$ are clearly revealed by the behavior of the averaged thermodynamic functions.

At any generation $G$, the partition function can be expressed in terms of the matrix elements of a $2 \times 2 \mathrm{TM} U_{G}$, after performing partial traces over all spins between $r_{1}$ and $r_{2}$. The self-similarity of lattice allows that traces are sequentially performed so that, at generation $G+1$, it is necessary only trace over the four independent partial traces of generation $G$.

For the low-dimensional matrices $U_{G}$ 's, such operations can be easily expressed in terms of the eigenvalues $\eta_{G}$ and $\epsilon_{G}$ of two subsequent generations, as can be easily demonstrated for the uniform ferromagnetic model. ${ }^{22}$ Moreover, in order to avoid numerical overflows of the Boltzmann 
weights for large values of $G$, the variable transformation

$$
\begin{gathered}
f_{G}=-N_{G}^{-1} T \ln \eta_{G}, \\
\xi_{G}=\frac{M_{G}}{\ln \left(\eta_{G} / \epsilon_{G}\right)},
\end{gathered}
$$

can be used in the program code to directly evaluate the free energy and correlation length at any value of $G$. In Eqs. (3) and (4), $N_{G}=2\left(2+4^{G}\right) / 3$ and $M_{G}=2^{G}$ denote, respectively, the number of spins and the chemical distance between the $r_{1}$ and $r_{2}$ at generation $G$. We refer to Ref. 15 for the explicit form of the maps for $\eta_{G}$ and $\epsilon_{G}$ as $f_{G+1}=f_{G+1}\left(f_{G}\right)$ and $\xi_{G+1}$ $=\xi_{G+1}\left(f_{G}, \xi_{G}\right)$ in the disordered case. Results for both the $\mathbf{L}$ and $\mathbf{C}$ models are obtained by the iteration of that same set of maps, provided we take into account the distribution expressed by Eq. (1), and the correct assignment of values of $J_{x}$ to each coupling constant.

Since the thermodynamic functions should be evaluated in the limit $G \rightarrow \infty$, we must introduce an approach to deal with intrinsic limitations of the used numeric method that restricts the evaluation of the properties of finite-size systems. As the number of independently coupling constants $J_{x}$ increases exponentially with $G$, we quickly exhaust the capacity of any computing system to date. Although we are forced to stop increasing the value of $G$, we can compare the series of average results obtained upon increasing the value of $G$, and check whether they are converging to $G$-independent value. This happens to be true for all values of $T$, although fluctuations become quite strong in the region close to $T_{c}$.

To warrant better results, it is convenient to use an approximate scheme introduced before. ${ }^{15}$ It amounts to store, in a data bank, the values of several function for a large number $N_{B}$ of independent samples. Each of them were evaluated by choosing random values of the coupling constants up to a value $G_{M}$. For any larger values of $G \in\left[G_{M}+1, G_{\infty}\right]$, the maps are iterated by randomly selecting four samples from the $G-1$ bank. Here, $G_{\infty}$ indicates the largest value of $G$ that we select to iterate the system using the sequences stored in the data banks.

To proceed with, all $G_{M}+\ell$ banks with $1 \leq \ell \leq G_{\infty}-G_{M}$ must also consist of $N_{B}$ samples, which are randomly assembled from samples of the $G_{M}+\ell-1$ bank. The difference in CPU time consuming with respect to the exact scheme is that the time dependence of the process is linear with respect to $G_{\infty}-G_{M}$. In the current study we consider $G_{\infty} \leq 60$ and $G_{M} \leq 11$, what amounts to consider, in the best case, more than $4 \times 10^{6}$ different values of $J_{x}$.

An important technical aspect of the work refers to the CPU time required to the evaluation of the $J_{x}$ series. Note that we have performed most of our work by taking $G_{M}$ $\geq 10$. Until this value of $G_{M}$, it is straightforward to evaluate the series $2^{G} \sim 10^{3}$ constants of the $\mathbf{L}$ model, where correlation is inserted along each consecutive shortest path linking the two root sites. However, the way the $J_{x}$ are inserted into the $\mathbf{C}$ model requires a much larger number of $J_{x}$ constants $\left(4^{G} \geq 10^{6}\right)$ in a single series. Since the number of operations required for the evaluation of Eq. (1) increases with $N^{2}$, and
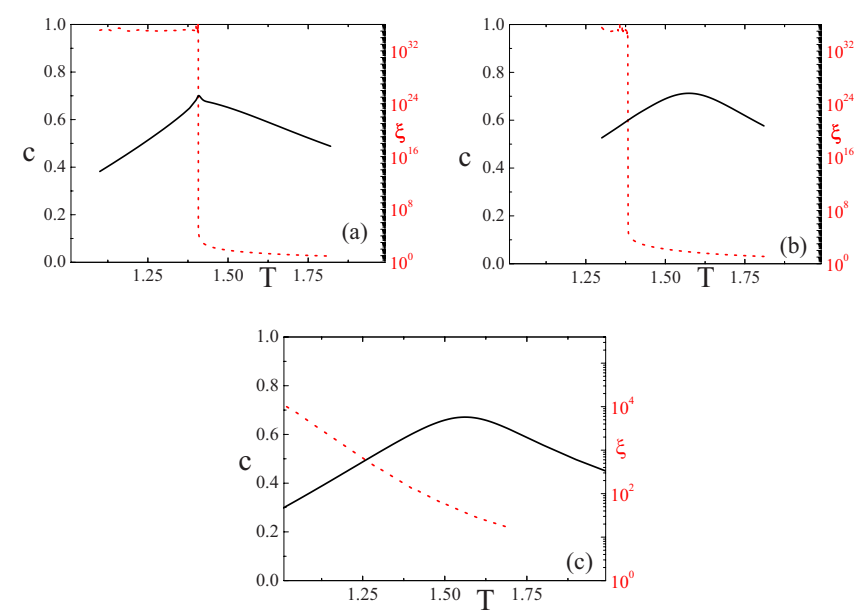

FIG. 2. (Color online) Plots of $\langle c\rangle$ (solid line) and $\langle\xi\rangle$ (dashed line) for $G_{M}=10$ and $G_{\infty}=60$. Values of $\langle c\rangle$ and $\langle\xi\rangle$ are indicated, respectively, on the left and right vertical axes. (a) $\mathbf{L}$ model, $\alpha$ $=1.5$, corresponding to region (III); (b) $\mathbf{C}$ model, $\alpha=1.5$, corresponding to region (II); (c) $\mathbf{C}$ model, $\alpha=1.4$, corresponding to region (I).

we have to generate a large number of independent series to build the coupling-constant bank, we approximated the $4^{10}$ series by the adding, in sequence, 64 independent series of length $4^{7}$. This decreases the CPU time to generate the required series by a factor 64 .

Other similar approaches have been used to infer properties of disordered systems in the thermodynamic limit, as several implementations of renormalization group in hierarchical structures. ${ }^{21,23,24}$ Any such approximate schemes introduces into the system spurious correlations since any bank for $G>G_{M}$ is built with the information of the $N_{B}$ samples at $G=G_{M}$. Nevertheless, the approximate schemes are reliable and contribute to reduce the relative size of fluctuations close to $T_{c}$.

\section{RESULTS}

Let us first discuss the behavior of $c$ and $\xi$ as function of $T$, displayed in Fig. 2, as they clearly indicate the changes in the ordering properties of the model. The curves correspond to average values $(\langle\cdot\rangle)$ of the respective quantities taken over individual samples, for fixed value of $\alpha$. For almost all values of $T$ good convergence (of the order $10^{-2}$ ) have been achieved by taking $G_{\infty}=G_{M}=10$. Only in a relatively close neighborhood of $T_{c}$ results for $G_{\infty}=60$ differ from those obtained for $G_{\infty}=10$.

To identify the existence of critical behavior we have paid attention to: (a) the existence of divergence in the value of $\xi$ as $T \rightarrow T_{c}$; (b) the formation of a cusp in $c\left(T=T_{c}\right)$. For both models, three different types of behavior have been identified, which depend on the increasing value of $\alpha$ : (I) neither (a) nor (b) are observed, i.e., $\xi$ seems to diverge only at $T$ $=0$ and $c(T)$ is always smooth. (II) (a) is observed at $T=T_{c}$ but $c\left(T_{c}\right)$ presents no singularity. (III) (a) and (b) occur at the same value $T=T_{c}$.

Examples of these typical behavior are displayed in Fig. 2, where we draw curves for $c_{G_{\infty}}$ and $\xi_{G_{\infty}}$ for the $\mathbf{L}$ model 

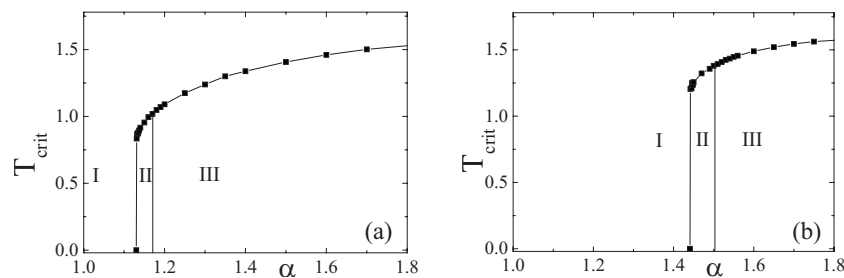

FIG. 3. Phase diagrams in the $(\alpha, T)$ plane, with indications of the regions (I), (II), and (III), for models (a) $\mathbf{L}$ and (b) $\mathbf{C}$. The corresponding values $\alpha_{c} \simeq 1.13$ and 1.44 are equivalent to the coordinate $p_{N}$ of the Nishimori points in the asymmetric bimodal probability distribution (Ref. 17).

when $\alpha=1.5$ [Fig. 2(a)], while in Fig. 2(b) we illustrate the behavior of the same quantities for the $\mathbf{C}$ model and same value of $\alpha$. We clearly see that model $\mathbf{L}$ already satisfies the conditions (III) but model $\mathbf{C}$ is in a region where condition (II) prevails. Finally, Fig. 2(c) corresponds to the situation of the $\mathbf{C}$ model where both $c$ and $\xi$ have a smooth behavior for all $T>0$.

The results obtained after scrutinizing the system in a wide region of values of $\alpha$ can be condensed in two phase diagrams in the $(\alpha, T)$ plane shown in Fig. 3 for $G_{M}=10$. They indicate that, for the $\mathbf{L}$ model, the regions (I), (II), and (III) are restricted, respectively, to the following intervals of values of $\alpha$ : $[0,1.13],[1.13,1.17]$, and $[1.17, \infty]$. The corresponding intervals for the $\mathbf{C}$ model are: [0,1.44], [1.44,1.51], and $[1.51, \infty]$. The diagrams are qualitatively similar to those found for the model of asymmetric distributions of values $\pm J$. For the sake of comparison, the corresponding threshold values $\alpha_{c}=1.13$ and 1.44 play similar roles that of $\mathbf{p}_{\mathbf{N}}$, the $\mathbf{p}$ coordinate of the Nishimori point in the asymmetric distribution model. However, to the best of our knowledge, there is no theoretical result equivalent to the NL to be added in the present case.

These results clearly show that the introduction of correlation between the coupling constants offer the system the possibility of developing ferromagnetic ordering. Note that, for both systems, the ordering appears at relative low values of $\alpha$ and $H$ in comparison, e.g., to observed values for electronic-state delocalization $(\alpha>2)$. Indeed, for systems with values of $H$ as low as 0.06 , which characterizes antipersistent correlation, critical behavior is observed.

It is interesting to comment on the effect of varying $G_{M}$ in the phase diagrams shown in Fig. 3. We have observed that the $\mathbf{C}$ model reaches convergence with respect to the value of $G_{M}$ much earlier than the $\mathbf{L}$ model. In the first case, our results for a fine scanning around $\alpha \sim 1.4$ for $G_{M}=9,10$, and 11 shows that the critical value $\alpha_{c}$ is reached, within $10^{-3}$ accuracy, already for $G_{M}=9$. The investigation of $\mathbf{L}$ model for $G_{M}=11$ indicates that the precision of quoted value of $\alpha_{c}$ is somewhat larger $\left(\sim 3 \times 10^{-2}\right)$. However, as the accuracy in the values of $\alpha_{c}$ has greatly improved when we compare with results for $G_{M}=8$ and $G_{M}=9$, we decided to provide results for both models we the same value of $G_{M}$.

Regions (I) and (III) can be associated to the situations where, respectively, the system can only be found at the paramagnetic phase for all values of $T$, or suffers a phase transition from the paramagnetic to the ferromagnetic phase.

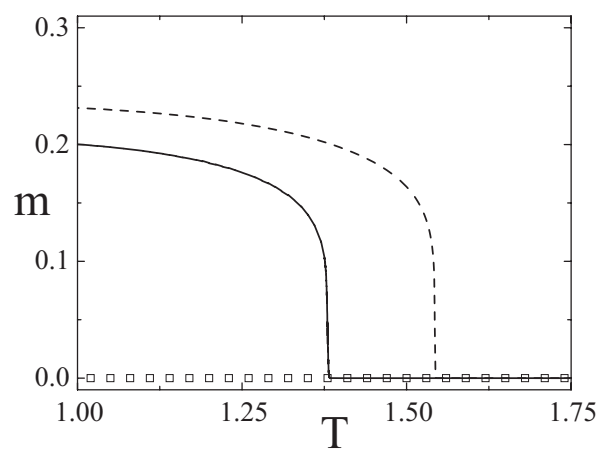

FIG. 4. Dependence of $m$ with respect to $T$ for three different values of $\alpha$, corresponding to the regions (I) (open squares), (II) (solid line), and (III) (dashed line). Only in region (I) the system does not show spontaneous magnetization.

On the other hand, if previous results clearly indicate that phase (II) has no equivalent in the asymmetric distributed model on a DHL for $p=b=2$, it is well known that similar models in dimensions higher than 2 do show more complex diagrams. In such cases, depending of the features of the model, spin-glass phases can be found in the intermediate region of values of $\mathbf{p}$. In particular, the transition from the paramagnetic to the spin-glass phase is characterized by a divergence in $\xi$ but no critical behavior in the specific heat. ${ }^{15}$

To elucidate the nature of the transition in the region II, we add a residual magnetic field acting on all spins. Proceeding this way, it is possible to adapt the TM method to evaluate the spontaneous magnetization $m$ per spin. This result provides a deeper insight into the kind of magnetic ordering that can be found in the model. In Fig. 4 we show how $m$ depends on $T$ for three different values of $\alpha$ for the $\mathbf{C}$ model. The curves clearly show that $m$ presents a critical behavior in both (II) and (III) regions, what discards the possibility to associate region (II) to a spin-glass-like frozen phase with zero magnetization. As expected, in the region (I) no magnetic ordering has been detected.

Note that, although magnetization is nonzero for $T<T_{c}$ for values of $\alpha$ in the regions (II) and (III), $m$ does not saturate to 1 . This suggests that magnetic ordering consists of many distinct domains that point in different directions. This observation is consistent with an overall picture that ordering occurs even when $H$ corresponds to an antipersistent behavior.

\section{CONCLUSIONS}

In this work we have investigated a disordered magnetic model where correlation between coupling constants can be tuned by an external parameter $\alpha$. This way, it is possible to continuously drive the system from a fully disordered to a ferromagnetic model. Our analysis shows the existence of a critical threshold value of $\alpha_{c}$ below which no ferromagnetic ordering is found at nonzero temperature. Above this threshold, the system undergoes a transition from the paramagnetic phase into a low-temperature phase with ferromagnetic order.

We have considered two different ways to introduce correlation into the nearest-neighbor interactions in the standard 
DHL. Although the qualitative behavior of both models are alike, quantitative differences indicate that if correlation is inserted along the different paths that link the DHL root nodes, the system is more likely to show ferromagnetic ordering at lower values of $\alpha$.

We have characterized such transition by the divergence of the correlation length as well as by vanishing of magnetization at a same critical temperature $T_{c}(\alpha)$. The behavior of the specific heat provides a further indication of critical behavior. However, the emergence of a well-characterized cusp for large value of $\alpha$ becomes a lot more weaker when $\alpha$ $\rightarrow \alpha_{c}$ from above.
This interesting model can be extended to DHL's with larger fractal dimensions, what can be easily done by increasing the number of parallel paths inserted between neighboring nodes. This can provide useful hints on the influence of correlations in actual disordered three-dimensional systems.

\section{ACKNOWLEDGMENT}

This work was partially supported by the Brazilian agency CNPq.
${ }^{1}$ K. Binder and A. P. Young, Rev. Mod. Phys. 58, 801 (1986).

${ }^{2}$ K. H. Fischer and J. A. Hertz, Spin Glasses (Cambridge University Press, Cambridge, 1991).

${ }^{3}$ Spin Glasses and Random Fields, edited by A. P. Young (World Scientific, Singapore, 1998).

${ }^{4}$ F. A. B. F. de Moura and M. L. Lyra, Phys. Rev. Lett. 81, 3735 (1998).

${ }^{5}$ F. M. Izrailev and A. A. Krokhin, Phys. Rev. Lett. 82, 4062 (1999).

${ }^{6}$ H. Shima, T. Nomura, and T. Nakayama, Phys. Rev. B 70, 075116 (2004).

${ }^{7}$ N. P. Greis and H. S. Greenside, Phys. Rev. A 44, 2324 (1991).

${ }^{8}$ R. P. A. Lima, M. L. Lyra, E. M. Nascimento, and A. D. de Jesus, Phys. Rev. B 65, 104416 (2002).

${ }^{9}$ M. H. Vainstein, R. Morgado, F. A. Oliveira, F. A. B. F. de Moura, and M. D. Coutinho-Filho, Phys. Lett. A 339, 33 (2005).

${ }^{10}$ F. A. B. F. de Moura, M. D. Coutinho-Filho, E. P. Raposo, and M. L. Lyra, Phys. Rev. B 68, 012202 (2003).

${ }^{11}$ A. N. Berker and S. Ostlund, J. Phys. C 12, 4961 (1979).
${ }^{12}$ M. Kaufman and R. B. Griffiths, Phys. Rev. B 24, 496 (1981).

${ }^{13}$ C. Tsallis and A. C. N. de Magalhães, Phys. Rep. 268, 305 (1996).

${ }^{14}$ L. Klein, J. Adler, A. Aharony, A. B. Harris, and Y. Meir, Phys. Rev. B 43, 11249 (1991).

${ }^{15}$ R. F. S. Andrade, E. Nogueira, Jr., and S. Coutinho, Phys. Rev. B 68, 104523 (2003).

${ }^{16}$ D. O. C. Santos, E. Nogueira, Jr., and R. F. S. Andrade, Phys. Rev. B 73, 174202 (2006).

${ }^{17}$ H. Nishimori, J. Phys. C 13, 4071 (1980).

${ }^{18}$ H. Nishimori, Prog. Theor. Phys. 66, 1169 (1981).

${ }^{19}$ H. Nishimori, Statistical Physics of Spin Glasses and Information Processing (Oxford University Press, Oxford, 2001).

${ }^{20}$ H. Nishimori, J. Phys. Soc. Jpn. 55, 3305 (1986).

${ }^{21}$ E. Nogueira, Jr., S. Coutinho, F. D. Nobre, and E. M. F. Curado, Physica A 271, 125 (1999).

${ }^{22}$ R. F. S. Andrade, Phys. Rev. E 61, 7196 (2000).

${ }^{23}$ B. W. Southern and A. P. Young, J. Phys. C 10, 2179 (1977).

${ }^{24}$ E. M. F. Curado and J. L. Meunier, Physica A 149, 164 (1988). 